\title{
Measurement of the Moments of the Hadronic Invariant Mass Distribution in Semileptonic $B$ Decays
}

D. Acosta, ${ }^{16}$ J. Adelman, ${ }^{12}$ T. Affolder,${ }^{9}$ T. Akimoto, ${ }^{54}$ M.G. Albrow,${ }^{15}$ D. Ambrose, ${ }^{43}$ S. Amerio, ${ }^{42}$ D. Amidei,${ }^{33}$ A. Anastassov ${ }^{50} \mathrm{~K}$. Anikeev, ${ }^{15}$ A. Annovi, ${ }^{44} \mathrm{~J}$. Antos, ${ }^{1}$ M. Aoki,${ }^{54}$ G. Apollinari, ${ }^{15}$ T. Arisawa, ${ }^{56} \mathrm{~J}-\mathrm{F}$. Arguin, ${ }^{32}$ A. Artikov, ${ }^{13}$ W. Ashmanskas, ${ }^{15}$ A. Attal, ${ }^{7}$ F. Azfar, ${ }^{41}$ P. Azzi-Bacchetta, ${ }^{42}$ N. Bacchetta, ${ }^{42} \mathrm{H}$. Bachacou, ${ }^{28}$ W. Badgett, ${ }^{15}$ A. Barbaro-Galtieri, ${ }^{28}$ G.J. Barker,${ }^{25}$ V.E. Barnes, ${ }^{46}$ B.A. Barnett, ${ }^{24}$ S. Baroiant, ${ }^{6}$ M. Barone, ${ }^{17}$ G. Bauer, ${ }^{31}$ F. Bedeschi, ${ }^{44}$ S. Behari, ${ }^{24}$ S. Belforte, ${ }^{53}$ G. Bellettini, ${ }^{44}$ J. Bellinger, ${ }^{58}$ E. Ben-Haim, ${ }^{15}$ D. Benjamin, ${ }^{14}$ A. Beretvas, ${ }^{15}$ A. Bhatti, ${ }^{48}$ M. Binkley, ${ }^{15}$ D. Bisello, ${ }^{42}$ M. Bishai, ${ }^{15}$ R.E. Blair, ${ }^{2}$ C. Blocker, ${ }^{5}$ K. Bloom, ${ }^{33}$

B. Blumenfeld, ${ }^{24}$ A. Bocci, ${ }^{48}$ A. Bodek, ${ }^{47}$ G. Bolla, ${ }^{46}$ A. Bolshov, ${ }^{31}$ P.S.L. Booth,${ }^{29}$ D. Bortoletto, ${ }^{46}$ J. Boudreau, ${ }^{45}$ S. Bourov, ${ }^{15}$ B. Brau,${ }^{9}$ C. Bromberg, ${ }^{34}$ E. Brubaker, ${ }^{12}$ J. Budagov, ${ }^{13}$ H.S. Budd, ${ }^{47}$ K. Burkett,${ }^{15}$ G. Busetto, ${ }^{42}$ P. Bussey, ${ }^{19}$ K.L. Byrum, ${ }^{2}$ S. Cabrera, ${ }^{14}$ M. Campanelli, ${ }^{18}$ M. Campbell, ${ }^{33}$ A. Canepa, ${ }^{46}$ M. Casarsa, ${ }^{53}$

D. Carlsmith, ${ }^{58}$ S. Carron, ${ }^{14}$ R. Carosi, ${ }^{44}$ M. Cavalli-Sforza, ${ }^{3}$ A. Castro, ${ }^{4}$ P. Catastini, ${ }^{44}$ D. Cauz, ${ }^{53}$ A. Cerri, ${ }^{28}$ L. Cerrito, ${ }^{23}$ J. Chapman, ${ }^{33}$ C. Chen,${ }^{43}$ Y.C. Chen, ${ }^{1}$ M. Chertok, ${ }^{6}$ G. Chiarelli, ${ }^{44}$ G. Chlachidze, ${ }^{13}$ F. Chlebana, ${ }^{15}$ I. Cho, ${ }^{27}$ K. Cho, ${ }^{27}$ D. Chokheli, ${ }^{13}$ J.P. Chou, ${ }^{20}$ M.L. Chu, ${ }^{1}$ S. Chuang, ${ }^{58}$ J.Y. Chung, ${ }^{38}$ W-H. Chung, ${ }^{58}$ Y.S. Chung, ${ }^{47}$ C.I. Ciobanu, ${ }^{23}$ M.A. Ciocci, ${ }^{44}$ A.G. Clark, ${ }^{18}$ D. Clark, ${ }^{5}$ M. Coca, ${ }^{47}$ A. Connolly, ${ }^{28}$ M. Convery, ${ }^{48}$

J. Conway, ${ }^{6}$ B. Cooper, ${ }^{30}$ M. Cordelli, ${ }^{17}$ G. Cortiana, ${ }^{42}$ J. Cranshaw, ${ }^{52}$ J. Cuevas, ${ }^{10}$ R. Culbertson, ${ }^{15}$ C. Currat, ${ }^{28}$ D. Cyr ${ }^{58}$ D. Dagenhart, ${ }^{5}$ S. Da Ronco, ${ }^{42}$ S. D'Auria, ${ }^{19}$ P. de Barbaro, ${ }^{47}$ S. De Cecco, ${ }^{49}$ G. De Lentdecker,${ }^{47}$

S. Dell'Agnello, ${ }^{17}$ M. Dell'Orso, ${ }^{44}$ S. Demers,${ }^{47}$ L. Demortier, ${ }^{48}$ M. Deninno, ${ }^{4}$ D. De Pedis,${ }^{49}$ P.F. Derwent,${ }^{15}$

C. Dionisi, ${ }^{49}$ J.R. Dittmann, ${ }^{15}$ C. Dörr,${ }^{25}$ P. Doksus,${ }^{23}$ A. Dominguez, ${ }^{28}$ S. Donati, ${ }^{44}$ M. Donega, ${ }^{18} \mathrm{~J}$. Donini, ${ }^{42}$ M. D'Onofrio, ${ }^{18}$ T. Dorigo, ${ }^{42}$ V. Drollinger, ${ }^{36}$ K. Ebina, ${ }^{56}$ N. Eddy, ${ }^{23}$ J. Ehlers, ${ }^{18}$ R. Ely, ${ }^{28}$ R. Erbacher, ${ }^{6}$ M. Erdmann, ${ }^{25}$ D. Errede, ${ }^{23}$ S. Errede, ${ }^{23}$ R. Eusebi, ${ }^{47}$ H.-C. Fang, ${ }^{28}$ S. Farrington, ${ }^{29}$ I. Fedorko, ${ }^{44}$ W.T. Fedorko, ${ }^{12}$ R.G. Feild, ${ }^{59}$ M. Feindt, ${ }^{25}$ J.P. Fernandez, ${ }^{46}$ C. Ferretti, ${ }^{33}$ R.D. Field, ${ }^{16}$ G. Flanagan, ${ }^{34}$ B. Flaugher ${ }^{15}$

L.R. Flores-Castillo, ${ }^{45}$ A. Foland,${ }^{20}$ S. Forrester, ${ }^{6}$ G.W. Foster, ${ }^{15}$ M. Franklin, ${ }^{20}$ J.C. Freeman, ${ }^{28}$ Y. Fujii, ${ }^{26}$

I. Furic, ${ }^{12}$ A. Gajjar, ${ }^{29}$ A. Gallas, ${ }^{37}$ J. Galyardt, ${ }^{11}$ M. Gallinaro, ${ }^{48}$ M. Garcia-Sciveres, ${ }^{28}$ A.F. Garfinkel, ${ }^{46}$

C. Gay, ${ }^{59}$ H. Gerberich, ${ }^{14}$ D.W. Gerdes,${ }^{33}$ E. Gerchtein, ${ }^{11}$ S. Giagu, ${ }^{49}$ P. Giannetti, ${ }^{44}$ A. Gibson, ${ }^{28}$

K. Gibson, ${ }^{11}$ C. Ginsburg, ${ }^{58}$ K. Giolo, ${ }^{46}$ M. Giordani, ${ }^{53}$ M. Giunta, ${ }^{44}$ G. Giurgiu, ${ }^{11}$ V. Glagolev, ${ }^{13}$

D. Glenzinski, ${ }^{15}$ M. Gold, ${ }^{36}$ N. Goldschmidt, ${ }^{33}$ D. Goldstein, ${ }^{7}$ J. Goldstein, ${ }^{41}$ G. Gomez, ${ }^{10}$ G. Gomez-Ceballos, ${ }^{31}$ M. Goncharov, ${ }^{51}$ O. González, ${ }^{46}$ I. Gorelov, ${ }^{36}$ A.T. Goshaw, ${ }^{14}$ Y. Gotra, ${ }^{45}$ K. Goulianos, ${ }^{48}$ A. Gresele, ${ }^{4}$

M. Griffiths, ${ }^{29}$ C. Grosso-Pilcher, ${ }^{12}$ U. Grundler,${ }^{23}$ M. Guenther, ${ }^{46}$ J. Guimaraes da Costa, ${ }^{20}$ C. Haber ${ }^{28}$ K. Hahn, ${ }^{43}$ S.R. Hahn, ${ }^{15}$ E. Halkiadakis, ${ }^{47}$ A. Hamilton, ${ }^{32}$ B-Y. Han,${ }^{47}$ R. Handler, ${ }^{58}$ F. Happacher, ${ }^{17}$ K. Hara,${ }^{54}$ M. Hare, ${ }^{55}$ R.F. Harr, ${ }^{57}$ R.M. Harris,${ }^{15}$ F. Hartmann, ${ }^{25}$ K. Hatakeyama, ${ }^{48}$ J. Hauser, ${ }^{7}$ C. Hays, ${ }^{14}$ H. Hayward, ${ }^{29}$ E. Heider,${ }^{55}$ B. Heinemann, ${ }^{29}$ J. Heinrich, ${ }^{43}$ M. Hennecke,${ }^{25}$ M. Herndon,${ }^{24}$ C. Hill, ${ }^{9}$ D. Hirschbuehl, ${ }^{25}$ A. Hocker,${ }^{47}$ K.D. Hoffman, ${ }^{12}$ A. Holloway, ${ }^{20}$ S. Hou,${ }^{1}$ M.A. Houlden,${ }^{29}$ B.T. Huffman, ${ }^{41}$ Y. Huang, ${ }^{14}$ R.E. Hughes, ${ }^{38}$

J. Huston, ${ }^{34}$ K. Ikado, ${ }^{56}$ J. Incandela, ${ }^{9}$ G. Introzzi, ${ }^{44}$ M. Iori, ${ }^{49}$ Y. Ishizawa, ${ }^{54}$ C. Issever, ${ }^{9}$ A. Ivanov, ${ }^{47}$ Y. Iwata, ${ }^{22}$ B. Iyutin, ${ }^{31}$ E. James, ${ }^{15}$ D. Jang, ${ }^{50}$ J. Jarrell, ${ }^{36}$ D. Jeans, ${ }^{49}$ H. Jensen, ${ }^{15}$ E.J. Jeon, ${ }^{27}$ M. Jones, ${ }^{46}$ K.K. Joo, ${ }^{27}$

S.Y. Jun, ${ }^{11}$ T. Junk, ${ }^{23}$ T. Kamon, ${ }^{51}$ J. Kang, ${ }^{33}$ M. Karagoz Unel, ${ }^{37}$ P.E. Karchin, ${ }^{57}$ S. Kartal, ${ }^{15}$ Y. Kato, ${ }^{40}$ Y. Kemp, ${ }^{25}$ R. Kephart, ${ }^{15}$ U. Kerzel, ${ }^{25}$ V. Khotilovich, ${ }^{51}$ B. Kilminster, ${ }^{38}$ D.H. Kim, ${ }^{27}$ H.S. Kim, ${ }^{23}$ J.E. Kim, ${ }^{27}$ M.J. Kim, ${ }^{11}$ M.S. Kim, ${ }^{27}$ S.B. Kim, ${ }^{27}$ S.H. Kim,${ }^{54}$ T.H. Kim, ${ }^{31}$ Y.K. Kim, ${ }^{12}$ B.T. King, ${ }^{29}$ M. Kirby, ${ }^{14}$ L. Kirsch, ${ }^{5}$ S. Klimenko, ${ }^{16}$ B. Knuteson, ${ }^{31}$ B.R. Ko, ${ }^{14}$ H. Kobayashi, ${ }^{54}$ P. Koehn, ${ }^{38}$ D. J. Kong, ${ }^{27}$ K. Kondo, ${ }^{56}$ J. Konigsberg, ${ }^{16}$ K. Kordas, ${ }^{32}$ A. Korn, ${ }^{31}$ A. Korytov, ${ }^{16}$ K. Kotelnikov, ${ }^{35}$ A.V. Kotwal, ${ }^{14}$ A. Kovalev, ${ }^{43}$ J. Kraus, ${ }^{23}$ I. Kravchenko, ${ }^{31}$ A. Kreymer, ${ }^{15}$ J. Kroll, ${ }^{43}$ M. Kruse, ${ }^{14}$ V. Krutelyov, ${ }^{51}$ S.E. Kuhlmann, ${ }^{2}$ S. Kwang, ${ }^{12}$ A.T. Laasanen, ${ }^{46}$ S. Lai, ${ }^{32}$

S. Lami, ${ }^{48}$ S. Lammel, ${ }^{15}$ J. Lancaster, ${ }^{14}$ M. Lancaster, ${ }^{30}$ R. Lander, ${ }^{6}$ K. Lannon, ${ }^{38}$ A. Lath, ${ }^{50}$ G. Latino, ${ }^{36}$

R. Lauhakangas, ${ }^{21}$ I. Lazzizzera, ${ }^{42}$ Y. Le,${ }^{24}$ C. Lecci,${ }^{25}$ T. LeCompte,${ }^{2}$ J. Lee, ${ }^{27}$ J. Lee, ${ }^{47}$ S.W. Lee, ${ }^{51}$ R. Lefèvre, ${ }^{3}$

N. Leonardo, ${ }^{31}$ S. Leone, ${ }^{44}$ S. Levy, ${ }^{12}$ J.D. Lewis, ${ }^{15}$ K. Li,${ }^{59}$ C. Lin, ${ }^{59}$ C.S. Lin, ${ }^{15}$ M. Lindgren, ${ }^{15}$ E. Lipeles,${ }^{8}$

T.M. Liss ${ }^{23}$ A. Lister, ${ }^{18}$ D.O. Litvintsev, ${ }^{15}$ T. Liu, ${ }^{15}$ Y. Liu, ${ }^{18}$ N.S. Lockyer, ${ }^{43}$ A. Loginov, ${ }^{35}$ M. Loreti, ${ }^{42}$

P. Loverre, ${ }^{49}$ R-S. Lu, ${ }^{1}$ D. Lucchesi, ${ }^{42}$ P. Lujan, ${ }^{28}$ P. Lukens, ${ }^{15}$ G. Lungu, ${ }^{16}$ L. Lyons, ${ }^{41}$ J. Lys ${ }^{28}$ R. Lysak, ${ }^{1}$

D. MacQueen, ${ }^{32}$ R. Madrak, ${ }^{15}$ K. Maeshima, ${ }^{15}$ P. Maksimovic, ${ }^{24}$ L. Malferrari, ${ }^{4}$ G. Manca, ${ }^{29}$ R. Marginean, ${ }^{38}$

C. Marino, ${ }^{23}$ A. Martin, ${ }^{24}$ M. Martin, ${ }^{59}$ V. Martin, ${ }^{37}$ M. Martínez, ${ }^{3}$ T. Maruyama, ${ }^{54}$ H. Matsunaga, ${ }^{54}$

M. Mattson, ${ }^{57}$ P. Mazzanti, ${ }^{4}$ K.S. McFarland, ${ }^{47}$ D. McGivern, ${ }^{30}$ P.M. McIntyre, ${ }^{51}$ P. McNamara, ${ }^{50}$ R. NcNulty, ${ }^{29}$ A. Mehta, ${ }^{29}$ S. Menzemer, ${ }^{31}$ A. Menzione, ${ }^{44}$ P. Merkel, ${ }^{15}$ C. Mesropian, ${ }^{48}$ A. Messina, ${ }^{49}$ T. Miao, ${ }^{15}$ N. Miladinovic,${ }^{5}$ L. Miller, ${ }^{20}$ R. Miller, ${ }^{34}$ J.S. Miller ${ }^{33}$ R. Miquel,${ }^{28}$ S. Miscetti, ${ }^{17}$ G. Mitselmakher,${ }^{16}$ A. Miyamoto, ${ }^{26}$ Y. Miyazaki, ${ }^{40}$ N. Moggi, ${ }^{4}$ B. Mohr, ${ }^{7}$ R. Moore,${ }^{15}$ M. Morello, ${ }^{44}$ P.A. Movilla Fernandez,${ }^{28}$ A. Mukherjee, ${ }^{15}$ M. Mulhearn, ${ }^{31}$ 
T. Muller, ${ }^{25}$ R. Mumford, ${ }^{24}$ A. Munar,${ }^{43}$ P. Murat,${ }^{15}$ J. Nachtman, ${ }^{15}$ S. Nahn, ${ }^{59}$ I. Nakamura,${ }^{43}$ I. Nakano, ${ }^{39}$ A. Napier, ${ }^{55}$ R. Napora,${ }^{24}$ D. Naumov ${ }^{36}$ V. Necula, ${ }^{16}$ F. Niell,${ }^{33}$ J. Nielsen, ${ }^{28}$ C. Nelson, ${ }^{15}$ T. Nelson, ${ }^{15}$ C. Neu, ${ }^{43}$ M.S. Neubauer,${ }^{8}$ C. Newman-Holmes, ${ }^{15}$ T. Nigmanov ${ }^{45}$ L. Nodulman, ${ }^{2}$ O. Norniella, ${ }^{3}$ K. Oesterberg, ${ }^{21}$ T. Ogawa, ${ }^{56}$ S.H. Oh, ${ }^{14}$ Y.D. Oh, ${ }^{27}$ T. Ohsugi, ${ }^{22}$ T. Okusawa,${ }^{40}$ R. Oldeman,${ }^{49}$ R. Orava,${ }^{21}$ W. Orejudos,${ }^{28}$ C. Pagliarone, ${ }^{44}$ E. Palencia ${ }^{10}$ R. Paoletti, ${ }^{44}$ V. Papadimitriou, ${ }^{15}$ S. Pashapour,${ }^{32}$ J. Patrick, ${ }^{15}$ G. Pauletta,${ }^{53}$ M. Paulini,${ }^{11}$ T. Pauly, ${ }^{41}$ C. Paus,${ }^{31}$ D. Pellett,${ }^{6}$ A. Penzo,${ }^{53}$ T.J. Phillips, ${ }^{14}$ G. Piacentino, ${ }^{44}$ J. Piedra,${ }^{10}$ K.T. Pitts, ${ }^{23}$ C. Plager,${ }^{7}$ A. Pompoš ${ }^{46}$ L. Pondrom,${ }^{58}$ G. Pope,${ }^{45}$ X. Portell,${ }^{3}$ O. Poukhov,${ }^{13}$ F. Prakoshyn, ${ }^{13}$ T. Pratt,${ }^{29}$ A. Pronko, ${ }^{16}$ J. Proudfoot,${ }^{2}$ F. Ptohos,${ }^{17}$ G. Punzi,${ }^{44}$ J. Rademacker ${ }^{41}$ M.A. Rahaman, ${ }^{45}$ A. Rakitine,${ }^{31}$ S. Rappoccio ${ }^{20}$ F. Ratnikov, ${ }^{50}$ H. Ray, ${ }^{33}$ B. Reisert,${ }^{15}$ V. Rekovic,${ }^{36}$ P. Renton, ${ }^{41}$ M. Rescigno, ${ }^{49}$ F. Rimondi, ${ }^{4}$ K. Rinnert,${ }^{25}$ L. Ristori,${ }^{44}$ W.J. Robertson, ${ }^{14}$ A. Robson, ${ }^{41}$ T. Rodrigo,${ }^{10}$ S. Rolli, ${ }^{55}$ L. Rosenson, ${ }^{31}$ R. Roser, ${ }^{15}$ R. Rossin, ${ }^{42}$ C. Rott, ${ }^{46}$ J. Russ, ${ }^{11}$ V. Rusu, ${ }^{12}$ A. Ruiz,${ }^{10}$ D. Ryan, ${ }^{55}$ H. Saarikko, ${ }^{21}$ S. Sabik, ${ }^{32}$ A. Safonov, ${ }^{6}$ R. St. Denis, ${ }^{19}$ W.K. Sakumoto, ${ }^{47}$ G. Salamanna, ${ }^{49}$ D. Saltzberg, ${ }^{7}$ C. Sanchez,${ }^{3}$ A. Sansoni, ${ }^{17}$ L. Santi, ${ }^{53}$ S. Sarkar ${ }^{49}$ K. Sato, ${ }^{54}$ P. Savard ${ }^{32}$ A. Savoy-Navarro, ${ }^{15}$ P. Schlabach, ${ }^{15}$ E.E. Schmidt,${ }^{15}$ M.P. Schmidt,${ }^{59}$ M. Schmitt ${ }^{37}$ L. Scodellaro, ${ }^{10}$ A. Scribano, ${ }^{44}$ F. Scuri,${ }^{44}$ A. Sedov, ${ }^{46}$ S. Seidel,${ }^{36}$ Y. Seiya,${ }^{40}$ F. Semeria,${ }^{4}$ L. Sexton-Kennedy, ${ }^{15}$ I. Sfiligoi, ${ }^{17}$ M.D. Shapiro, ${ }^{28}$ T. Shears,${ }^{29}$ P.F. Shepard,${ }^{45}$ D. Sherman,${ }^{20}$ M. Shimojima, ${ }^{54}$ M. Shochet, ${ }^{12}$ Y. Shon, ${ }^{58}$ I. Shreyber, ${ }^{35}$ A. Sidoti, ${ }^{44}$ J. Siegrist,${ }^{28}$ M. Siket,${ }^{1}$ A. Sill,${ }^{52}$ P. Sinervo, ${ }^{32}$ A. Sisakyan, ${ }^{13}$ A. Skiba, ${ }^{25}$ A.J. Slaughter ${ }^{15}$ K. Sliwa, ${ }^{55}$ D. Smirnov, ${ }^{36}$ J.R. Smith, ${ }^{6}$ F.D. Snider, ${ }^{15}$ R. Snihur, ${ }^{32}$ A. Soha, ${ }^{6}$ S.V. Somalwar, ${ }^{50}$ J. Spalding, ${ }^{15}$ M. Spezziga,${ }^{52}$ L. Spiegel,${ }^{15}$ F. Spinella,${ }^{44}$ M. Spiropulu, ${ }^{9}$ P. Squillacioti,${ }^{44}$ H. Stadie, ${ }^{25}$ B. Stelzer,${ }^{32}$ O. Stelzer-Chilton, ${ }^{32}$ J. Strologas,${ }^{36}$ D. Stuart, ${ }^{9}$ A. Sukhanov, ${ }^{16}$ K. Sumorok,${ }^{31}$ H. Sun, ${ }^{55}$ T. Suzuki,${ }^{54}$ A. Taffard,${ }^{23}$ R. Tafirout ${ }^{32}$ S.F. Takach, ${ }^{57}$ H. Takano, ${ }^{54}$ R. Takashima,${ }^{22}$ Y. Takeuchi, ${ }^{54}$ K. Takikawa, ${ }^{54}$ M. Tanaka,${ }^{2}$ R. Tanaka ${ }^{39}$ N. Tanimoto,${ }^{39}$ S. Tapprogge,${ }^{21}$ M. Tecchio, ${ }^{33}$ P.K. Teng, ${ }^{1}$ K. Terashi, ${ }^{48}$ R.J. Tesarek, ${ }^{15}$ S. Tether, ${ }^{31}$ J. Thom, ${ }^{15}$ A.S. Thompson, ${ }^{19}$ E. Thomson, ${ }^{43}$ P. Tipton,${ }^{47}$ V. Tiwari, ${ }^{11}$ S. Tkaczyk,${ }^{15}$ D. Toback, ${ }^{51}$ K. Tollefson, ${ }^{34}$ T. Tomura,${ }^{54}$ D. Tonelli, ${ }^{44}$ M. Tönnesmann, ${ }^{34}$ S. Torre, ${ }^{44}$ D. Torretta,${ }^{15}$ S. Tourneur, ${ }^{15}$ W. Trischuk,${ }^{32}$ J. Tseng, ${ }^{41}$ R. Tsuchiya,${ }^{56}$ S. Tsuno, ${ }^{39}$ D. Tsybychev,${ }^{16}$ N. Turini, ${ }^{44}$ M. Turner, ${ }^{29}$ F. Ukegawa,${ }^{54}$ T. Unverhau ${ }^{19}$ S. Uozumi, ${ }^{54}$ D. Usynin,${ }^{43}$ L. Vacavant, ${ }^{28}$ A. Vaiciulis, ${ }^{47}$ A. Varganov ${ }^{33}$ E. Vataga,${ }^{44}$ S. Vejcik III,${ }^{15}$ G. Velev ${ }^{15}$ V. Veszpremi, ${ }^{46}$ G. Veramendi, ${ }^{23}$ T. Vickey, ${ }^{23}$ R. Vidal, ${ }^{15}$ I. Vila, ${ }^{10}$ R. Vilar, ${ }^{10}$ I. Vollrath, ${ }^{32}$ I. Volobouev, ${ }^{28}$ M. von der Mey, ${ }^{7}$ P. Wagner, ${ }^{51}$ R.G. Wagner,${ }^{2}$ R.L. Wagner, ${ }^{15}$ W. Wagner,${ }^{25}$ R. Wallny, ${ }^{7}$ T. Walter, ${ }^{25}$ T. Yamashita, ${ }^{39}$ K. Yamamoto, ${ }^{40}$ Z. Wan, ${ }^{50}$ M.J. Wang, ${ }^{1}$ S.M. Wang, ${ }^{16}$ A. Warburton, ${ }^{32}$ B. Ward,${ }^{19}$ S. Waschke, ${ }^{19}$ D. Waters, ${ }^{30}$ T. Watts,${ }^{50}$ M. Weber, ${ }^{28}$ W.C. Wester III,${ }^{15}$ B. Whitehouse, ${ }^{55}$ A.B. Wicklund, ${ }^{2}$ E. Wicklund, ${ }^{15}$ H.H. Williams, ${ }^{43}$ P. Wilson, ${ }^{15}$ B.L. Winer, ${ }^{38}$ P. Wittich,${ }^{43}$ S. Wolbers,${ }^{15}$ M. Wolter,${ }^{55}$

M. Worcester, ${ }^{7}$ S. Worm ${ }^{50}$ T. Wright, ${ }^{33}$ X. Wu ${ }^{18}$ F. Würthwein, ${ }^{8}$ A. Wyatt, ${ }^{30}$ A. Yagil, ${ }^{15}$ C. Yang, ${ }^{59}$ U.K. Yang, ${ }^{12}$ W. Yao, ${ }^{28}$ G.P. Yeh, ${ }^{15}$ K. Yi, ${ }^{24}$ J. Yoh, ${ }^{15}$ P. Yoon, ${ }^{47}$ K. Yorita, ${ }^{56}$ T. Yoshida, ${ }^{40}$ I. Yu ${ }^{27}$ S. Yu ${ }^{43}$ Z. Yu, ${ }^{59}$ J.C. Yun, ${ }^{15}$ L. Zanello, ${ }^{49}$ A. Zanetti, ${ }^{53}$ I. Zaw ${ }^{20}$ F. Zetti, ${ }^{44}$ J. Zhou, ${ }^{50}$ A. Zsenei, ${ }^{18}$ and S. Zucchelli ${ }^{4}$

\section{(CDF Collaboration)}

1 Institute of Physics, Academia Sinica, Taipei, Taiwan 11529, Republic of China

2 Argonne National Laboratory, Argonne, Illinois 60439

3 Institut de Fisica d'Altes Energies, Universitat Autonoma de Barcelona, E-08193, Bellaterra (Barcelona), Spain

4 Istituto Nazionale di Fisica Nucleare, University of Bologna, I-40127 Bologna, Italy

5 Brandeis University, Waltham, Massachusetts 02254

6 University of California at Davis, Davis, California 95616

7 University of California at Los Angeles, Los Angeles, California 90024

8 University of California at San Diego, La Jolla, California 92093

9 University of California at Santa Barbara, Santa Barbara, California 93106

10 Instituto de Fisica de Cantabria, CSIC-University of Cantabria, 39005 Santander, Spain

11 Carnegie Mellon University, Pittsburgh, PA 15213

12 Enrico Fermi Institute, University of Chicago, Chicago, Illinois 60637

13 Joint Institute for Nuclear Research, RU-141980 Dubna, Russia

14 Duke University, Durham, North Carolina 27708

15 Fermi National Accelerator Laboratory, Batavia, Illinois 60510

16 University of Florida, Gainesville, Florida 32611

17 Laboratori Nazionali di Frascati, Istituto Nazionale di Fisica Nucleare, I-00044 Frascati, Italy 
18 University of Geneva, CH-1211 Geneva 4, Switzerland

19 Glasgow University, Glasgow G12 8QQ, United Kingdom

20 Harvard University, Cambridge, Massachusetts 02138

21 The Helsinki Group: Helsinki Institute of Physics; and Division of High Energy Physics,

Department of Physical Sciences, University of Helsinki, FIN-00044, Helsinki, Finland

22 Hiroshima University, Higashi-Hiroshima 724, Japan

23 University of Illinois, Urbana, Illinois 61801

24 The Johns Hopkins University, Baltimore, Maryland 21218

25 Institut für Experimentelle Kernphysik, Universität Karlsruhe, 76128 Karlsruhe, Germany

26 High Energy Accelerator Research Organization (KEK), Tsukuba, Ibaraki 305, Japan

27 Center for High Energy Physics: Kyungpook National University, Taegu 702-701; Seoul

National University, Seoul 151-742; and SungKyunKwan University, Suwon 440-746; Korea

28 Ernest Orlando Lawrence Berkeley National Laboratory, Berkeley, California 94720

29 University of Liverpool, Liverpool L69 7ZE, United Kingdom

30 University College London, London WC1E 6BT, United Kingdom

31 Massachusetts Institute of Technology, Cambridge, Massachusetts 02139

32 Institute of Particle Physics: McGill University, Montréal, Canada H3A 2T8; and University of Toronto, Toronto, Canada M5S 1A7

33 University of Michigan, Ann Arbor, Michigan 48109

34 Michigan State University, East Lansing, Michigan 48824

35 Institution for Theoretical and Experimental Physics, ITEP, Moscow 117259, Russia

36 University of New Mexico, Albuquerque, New Mexico 87131

37 Northwestern University, Evanston, Illinois 60208

38 The Ohio State University, Columbus, Ohio 43210

39 Okayama University, Okayama 700-8530, Japan

40 Osaka City University, Osaka 588, Japan

41 University of Oxford, Oxford OX1 3RH, United Kingdom

42 University of Padova, Istituto Nazionale di Fisica Nucleare, Sezione di Padova-Trento, I-35131 Padova, Italy

43 University of Pennsylvania, Philadelphia, Pennsylvania 19104

44 Istituto Nazionale di Fisica Nucleare, University and Scuola Normale Superiore of Pisa, I-56100 Pisa, Italy

45 University of Pittsburgh, Pittsburgh, Pennsylvania 15260

46 Purdue University, West Lafayette, Indiana 47907

47 University of Rochester, Rochester, New York 14627

48 The Rockefeller University, New York, New York 10021

49 Istituto Nazionale di Fisica Nucleare, Sezione di Roma 1, University di Roma "La Sapienza," I-00185 Roma, Italy

50 Rutgers University, Piscataway, New Jersey 08855

51 Texas A\&M University, College Station, Texas 77843

52 Texas Tech University, Lubbock, Texas 79409

53 Istituto Nazionale di Fisica Nucleare, University of Trieste/ Udine, Italy

54 University of Tsukuba, Tsukuba, Ibaraki 305, Japan

55 Tufts University, Medford, Massachusetts 02155

56 Waseda University, Tokyo 169, Japan

57 Wayne State University, Detroit, Michigan 48201

58 University of Wisconsin, Madison, Wisconsin 53706

59 Yale University, New Haven, Connecticut 06520

(Dated: March 15, 2005)

Using $180 \mathrm{pb}^{-1}$ of data collected with the CDF II detector at the Tevatron, we measure the first two moments of the hadronic invariant mass-squared distribution in charmed semileptonic $B$ decays. From these we determine the non-perturbative Heavy Quark Effective Theory parameters $\Lambda$ and $\lambda_{1}$ used to relate the $B$ meson semileptonic branching ratio to the CKM matrix element $\left|V_{c b}\right|$. For a minimum lepton momentum of $0.7 \mathrm{GeV} / c$ in the $B$ rest frame we measure the first two moments of the $D^{* *} \rightarrow D^{(*)} \pi$ component to be $\left\langle m_{D^{* *}}^{2}\right\rangle=\left(5.83 \pm 0.16_{\text {stat }} \pm 0.08_{\text {syst }}\right) \mathrm{GeV}^{2} / c^{4}$ and $\left\langle\left(m_{D^{* *}}^{2}-\left\langle m_{D^{* *}}^{2}\right\rangle\right)^{2}\right\rangle=\left(1.30 \pm 0.69_{\text {stat }} \pm 0.22_{\text {syst }}\right) \mathrm{GeV}^{4} / c^{8}$. Combining these with the discrete mass terms from the $D$ and $D^{*}$ mesons, we find the total moments to be $\left\langle M_{X_{c}}^{2}\right\rangle-\bar{m}_{D}^{2}=(0.467 \pm$ $\left.0.038_{\text {stat }} \pm 0.068_{\text {syst }}\right) \mathrm{GeV}^{2} / c^{4}$ and $\left\langle\left(M_{X_{c}}^{2}-\left\langle M_{X_{c}}^{2}\right\rangle\right)^{2}\right\rangle=\left(1.05 \pm 0.26_{\text {stat }} \pm 0.13_{\text {syst }}\right) \mathrm{GeV}^{4} / c^{8}$, where $\bar{m}_{D}$ is the spin-averaged $D$ mass. The systematic error is dominated by the uncertainties in the 
world-average branching ratios used to combine the $D, D^{*}$, and $D^{* *}$ contributions. The analysis makes no assumptions about the shape or resonant structure of the $D^{* *} \rightarrow D^{(*)} \pi$ invariant mass distribution.

PACS numbers: $\quad$ 13.20.He, $12.15 . \mathrm{Hh}, 12.39 . \mathrm{Hg}$

\section{INTRODUCTION}

In order to constrain the length of the side opposite the angle $\beta$ in the Cabibbo-Kobayashi-Maskawa (CKM) unitarity triangle, a precise measurement of the ratio $\left|V_{u b}\right| /\left|V_{c b}\right|$ is needed. The matrix element $\left|V_{c b}\right|$ is generally extracted from semileptonic $B$ decays. Currently, the most precise method is based on the measurement of the inclusive semileptonic partial width into charm, $\Gamma_{s l}=\Gamma\left(B \rightarrow X_{c} l \nu_{l}\right)$. The Operator Product Expansion (OPE) applied to Heavy Quark Effective Theory (HQET) relates the experimental determination of $\Gamma_{s l}$ to $\left|V_{c b}\right|$ 1, 2]. The relationship takes the form of an expansion in inverse powers of the $B$ mass, $m_{B}$. At each order in the expansion, new free non-perturbative parameters enter: one $(\Lambda)$ at order $1 / m_{B}$, two $\left(\lambda_{1}\right.$ and $\left.\lambda_{2}\right)$ at order $1 / m_{B}^{2}$, six at order $1 / m_{B}^{3}$, etc. In order to extract $\left|V_{c b}\right|$ from $\Gamma_{s l}$ some external information on these parameters is needed.

The same theoretical framework that predicts the value of $\Gamma_{s l}$ predicts the value of any weighted integral of the differential rate $d \Gamma_{s l} / d s_{H}$, provided the weight is a smooth function of $s_{H} \equiv M_{X_{c}}^{2}$. Using weight functions $\left(s_{H}-\bar{m}_{D}^{2}\right)$ and $\left(s_{H}-\left\langle s_{H}\right\rangle\right)^{2}$, with $\bar{m}_{D}=0.25 m_{D}+$ $0.75 m_{D^{*}}$, one can define the first two moments of the hadronic mass distribution:

$$
\begin{aligned}
& M_{1}=\int_{s_{H}^{\text {min }}}^{s_{H}^{\max }} d s_{H}\left(s_{H}-\bar{m}_{D}^{2}\right) \frac{1}{\Gamma_{s l}} \frac{d \Gamma_{s l}}{d s_{H}}, \\
& M_{2}=\int_{s_{H}^{\text {min }}}^{s_{H}^{\max }} d s_{H}\left(s_{H}-\left\langle s_{H}\right\rangle\right)^{2} \frac{1}{\Gamma_{s l}} \frac{d \Gamma_{s l}}{d s_{H}},
\end{aligned}
$$

which are simply the shifted mean and variance of the $M_{X_{c}}^{2}$ distribution in semileptonic charmed decays of $B$ mesons. The moments are not sensitive to $\left|V_{c b}\right|$, but they are more sensitive to the non-perturbative parameters of HQET than $\Gamma_{s l}$ itself is. Therefore, measuring the moments provides a useful constraint on the HQET parameters which improves the overall precision on $\left|V_{c b}\right|$ as determined from $\Gamma_{s l}$. This is the purpose of this analysis. Since $\lambda_{2}$ is well determined from the values of the hyperfine mass splittings in the $B$ and $D$ meson systems [2], only $\Lambda$ and $\lambda_{1}$ are studied here.

The $s_{H}$ distribution in $B^{-} \rightarrow X_{c}^{0} l^{-} \bar{\nu}_{l}$ decays can be split into three contributions corresponding to $X_{c}^{0}=$ $D^{0}, D^{* 0}, D^{* * 0}$. Here $D^{* * 0}$ stands for any neutral charmed state, resonant or not, other than $D^{0}, D^{* 0}$. The differ- ential mass-squared spectrum can be written as:

$$
\begin{aligned}
\frac{1}{\Gamma_{s l}} \frac{d \Gamma_{s l}}{d s_{H}} & =\frac{\Gamma_{0}}{\Gamma_{s l}} \cdot \delta\left(s_{H}-m_{D^{0}}^{2}\right)+\frac{\Gamma_{*}}{\Gamma_{s l}} \cdot \delta\left(s_{H}-m_{D^{* 0}}^{2}\right) \\
& +\left(1-\frac{\Gamma_{0}}{\Gamma_{s l}}-\frac{\Gamma_{*}}{\Gamma_{s l}}\right) \cdot f^{* *}\left(s_{H}\right),
\end{aligned}
$$

where $\Gamma_{s l}$ is now the inclusive $B^{-}$semileptonic width, $\Gamma_{0}$ and $\Gamma_{*}$ are the exclusive $B^{-}$partial widths to $D^{0} l^{-} \bar{\nu}_{l}$ and $D^{* 0} l^{-} \bar{\nu}_{l}$ respectively, and $f^{* *}\left(s_{H}\right)$ is the normalized hadronic invariant mass-squared distribution in the $D^{* * 0}$ channel. We use world-average values of $\Gamma_{0} / \Gamma_{s l}$, $\Gamma_{*} / \Gamma_{s l}, m_{D^{0}}$ and $m_{D^{* 0}}$ from the Particle Data Group [3] and concentrate on measuring $f^{* *}\left(s_{H}\right)$. In this way, we have only to measure the invariant mass distribution for the $D^{* * 0}$ component without having to determine the $D^{0}, D^{* 0}$ components or the relative normalizations between those and the $D^{* * 0}$ channel. The $D^{* * 0}$ spectrum is not well known, and includes, at least, two narrow and two wide states, together with a possible non-resonant $D^{(*)} \mathrm{n} \pi$ contribution. The measurement of the $D^{* * 0}$ spectrum is the main task of this analysis. We assume that the $D^{(*)} \pi l^{-} \bar{\nu}_{l}$ decays of $B^{-}$saturate the difference between its inclusive semileptonic decay rate and the sum of its exclusive decay rates to $D^{0} l^{-} \bar{\nu}_{l}$ and $D^{* 0} l^{-} \bar{\nu}_{l}$. We neglect all modes with additional pions in the final state, as well as $D^{* * 0} \rightarrow D_{s}^{(*)+} K^{-}$.

Only $D^{(*)+} \pi^{-}$decays (charge conjugated channels are implicitly included throughout the paper) are reconstructed. Contributions to the $s_{H}$ distribution from decays with neutral particles are included by applying isospin factors to the charged modes. Feed-down from one channel to another due to unmeasured neutral particles is subtracted statistically using the data themselves and isospin relations, as explained in Section 11 II

\section{DATA ANALYSIS}

The analysis uses a data sample of $\bar{p} p$ collisions at $\sqrt{s}=1.96 \mathrm{TeV}$ with an integrated luminosity of about $180 \mathrm{pb}^{-1}$, collected between February 2002 and August 2003 with the upgraded Collider Detector at the Fermilab Tevatron (CDF II). A description of the detector can be found in [4]. The relevant components for this analysis include a tracking system composed of a silicon strip vertex detector (SVX II) surrounded by an open cell drift chamber system (COT). The SVX II detector comprises five concentric layers of double-sided sensors located at radii between 2.5 and $10.6 \mathrm{~cm}$, while the COT provides 96 measurements (including axial and stereo) out to a radius of $132 \mathrm{~cm}$. The central tracking system is immersed in a $1.4 \mathrm{~T}$ solenoidal magnetic field. 
Two sampling calorimeters surround the magnetic coil. A set of proportional chambers inside the electromagnetic calorimeter provides information on the shower profile for use in electron identification. Muon candidates are identified with two sets of multi-layer drift chambers, one located outside the calorimeters and the other behind an additional $60 \mathrm{~cm}$-thick iron shield.

Decays $B \rightarrow D^{(*)+} \pi^{-} l^{-} X$, where $l$ stands for electron or muon, were recorded using a trigger that requires a lepton $l$ and a track displaced from the interaction point [5]. The lepton and the displaced track must have transverse momentum $p_{T}$ in excess of $4 \mathrm{GeV} / c$ and $2 \mathrm{GeV} / c$ respectively. The displaced track's impact parameter with respect to the beamline has to exceed $120 \mu \mathrm{m}$ and be below $1 \mathrm{~mm}$. Events which pass the trigger are recorded for further analysis. Only wellreconstructed tracks with $p_{T} \geq 0.4 \mathrm{GeV} / c$ are retained. Track parameters are corrected for the ionization energy loss appropriate to the mass hypothesis under consideration. A Monte Carlo sample of $B \rightarrow D^{* *} l \nu_{l}$ events based on the ISGW2 [6] and Goity-Roberts [] matrix elements and including a detailed simulation of the CDF II detector based on the GEANT [8] package has been used throughout the analysis. In accordance with our assumption, only $D^{* *} \rightarrow D^{(*)} \pi$ decays are generated.

Events with $D^{*+}\left(\rightarrow D^{0} \pi_{*}^{+}\right) l^{-}$and $D^{+} l^{-}$combinations are reconstructed in the decay channels $D^{0} \rightarrow$ $K^{-} \pi^{+}, K^{-} \pi^{+} \pi^{-} \pi^{+}, K^{-} \pi^{+} \pi^{0}$ and $D^{+} \rightarrow K^{-} \pi^{+} \pi^{+}$. Tracks with the appropriate charge combination are required to be consistent with a common vertex in three dimensions. One of the tracks in the vertex must fulfill the displaced trigger requirements. Suitable ranges are selected in the $D^{0}\left(1.84-1.89 \mathrm{GeV} / c^{2}\right)$ and $D^{+}(1.84-$ $\left.1.89 \mathrm{GeV} / c^{2}\right)$ mass distributions. For the $D^{*+}$ channel, an additional charged track $\left(\pi_{*}^{+}\right)$is required, such that the $M\left(D^{0} \pi_{*}^{+}\right)-M\left(D^{0}\right)$ mass difference lies between 0.142 and $0.147^{*} \mathrm{GeV} / c^{2}$. The $D^{0} \rightarrow K^{-} \pi^{+} \pi^{0}$ channel is reconstructed from the satellite peak in the $K^{-} \pi^{+}$ mass distribution $\left(1.50-1.70 \mathrm{GeV} / c^{2}\right)$. In this case the $M\left(K^{-} \pi^{+} \pi_{*}^{+}\right)-M\left(K^{-} \pi^{+}\right)$mass difference is required to be between 0.142 and $0.155 \mathrm{GeV} / c^{2}$. Duplicate removal is performed in the $D^{0} \rightarrow K^{-} \pi^{+} \pi^{-} \pi^{+}$channel: when two $D^{*+}$ candidates share all five tracks, differing only in the kaon mass assignment, the candidate with the $K^{-} \pi^{+} \pi^{-} \pi^{+}$mass closer to the nominal $D^{0}$ mass is retained. No attempt has been made to further identify kaons and pions.

After the selection of $D^{*+} l^{-}$and $D^{+} l^{-}$combinations, we obtain $3890 \pm 63,2994 \pm 57,6638 \pm 98$ and $14416 \pm 202$ signal events in the $K^{-} \pi^{+}, K^{-} \pi^{+} \pi^{-} \pi^{+}$, $K^{-} \pi^{+} \pi^{0}$ and $K^{-} \pi^{+} \pi^{+}$channels, respectively. Combinatorial backgrounds, estimated from sidebands of the $M\left(D^{+}\right)$and $M\left(D^{0} \pi_{*}^{+}\right)-M\left(D^{0}\right)$ distributions, have been subtracted. The quoted yield in the $D^{+}$channel has been rescaled by a factor 0.96 to account for the background from $D_{s}^{+} \rightarrow K^{+} K^{-} \pi^{+}$decays where the $K^{+}$is assigned the pion mass. Figure 1 shows on the left the $M\left(D^{0} \pi_{*}^{+}\right)-M\left(D^{0}\right)$ distributions for $D^{0}$ decaying into either $K^{-} \pi^{+}$or $K^{-} \pi^{+} \pi^{-} \pi^{+}$, and for $D^{0} \rightarrow K^{-} \pi^{+} \pi^{0}$, while the $D^{+} \rightarrow K^{-} \pi^{+} \pi^{+}$mass distribution is plotted on the right.

The $D^{(*)+} l^{-}$vertex (the $B$ vertex) is reconstructed in three dimensions and required to be at least $500 \mu \mathrm{m}$ away from the beam line. An additional pion $\left(\pi_{* *}^{-}\right)$is then added to create full $D^{+(*)} \pi^{-} l^{-}$candidates. The $\pi_{* *}^{-}$'s trajectory is required to be at most 2.5 standard deviations away from the $B$ vertex, and at least three standard deviations away from the beam line. These cuts were optimized using the $B \rightarrow D^{* *} l \nu_{l}$ Monte Carlo for the signal, and wrong-sign $\pi_{* *}^{+} l^{-}$combinations in data for the background. The measured mass distributions in the $D^{*+} \pi_{* *}$ and $D^{+} \pi_{* *}$ channels are shown in Fig. 2

\section{BACKGROUND AND EFFICIENCY CORRECTIONS}

The most important background sources are combinatorial background under the $D^{+}$and $D^{*}$ mass peaks, prompt tracks (from fragmentation or the underlying event) that fake $\pi_{* *}$ candidates, and feed-down from $D^{*+} \rightarrow D^{+} \pi^{0}$ decays into the $D^{+}$channel. Data sidebands are used to assess combinatorial background under the $D^{+}$mass and $D^{*+}{ }_{-} D^{0}$ mass-difference peaks, and wrong-sign $\pi_{* *}^{+} l^{-}$combinations in data to characterize the prompt background to the $\pi_{* *}$ candidates. The wrong-sign pion-lepton sample is subtracted from the right-sign sample, after performing side-band subtraction in both. A possible difference between the rate of prompt background with the right and the wrong $\pi_{* *}$ charge has been studied with a sample of fully reconstructed $B$ decays $\left(B^{-} \rightarrow J / \Psi K^{-}, B^{0} \rightarrow D^{(*)-} \pi^{+}, B^{-} \rightarrow D^{0} \pi^{-}\right)$ and found to be at most $4 \%$. This has been included in the systematic error. The bias in the background subtraction introduced by using the same wrong-sign sample for both the optimization of the selection and the final background subtraction has been studied using bootstrap [9] copies of the data and found to be smaller than $15 \%$ of the statistical error on the moments. This upper bound on the bias has been added as an additional systematical error.

Since we do not reconstruct neutral particles, events with a decay $B^{-} \rightarrow D^{*+} \pi_{* *}^{-} l^{-} \bar{\nu}_{l}$ with the $D^{*+}$ decaying into $D^{+} \pi^{0}$ constitute an irreducible background to the signal channel $B^{-} \rightarrow D^{+} \pi_{* *}^{-} l^{-} \bar{\nu}_{l}$. Using isospin symmetry, the background rate and $D^{+} \pi_{* *}^{-}$invariant mass can be obtained from the $D^{0} \pi_{* *}^{-}$invariant mass in $B^{-} \rightarrow D^{*+} \pi_{* *}^{-} l^{-} \bar{\nu}_{l}$ decays with $D^{*+} \rightarrow D^{0} \pi_{*}^{+}$, which we fully reconstruct, after correcting for the relative efficiency using Monte Carlo. A small physics background (around 1\% in rate), coming mostly from $B \rightarrow D_{s}^{(*)} D^{(*)}$ decays with the $D_{s}^{(*)}$ decaying semileptonically, is subtracted using Monte Carlo predictions. Background from tracks faking a lepton has been studied by looking at wrong-sign $D^{(*)+} l^{+}$combinations. It has been found that the background subtraction procedure outlined above 

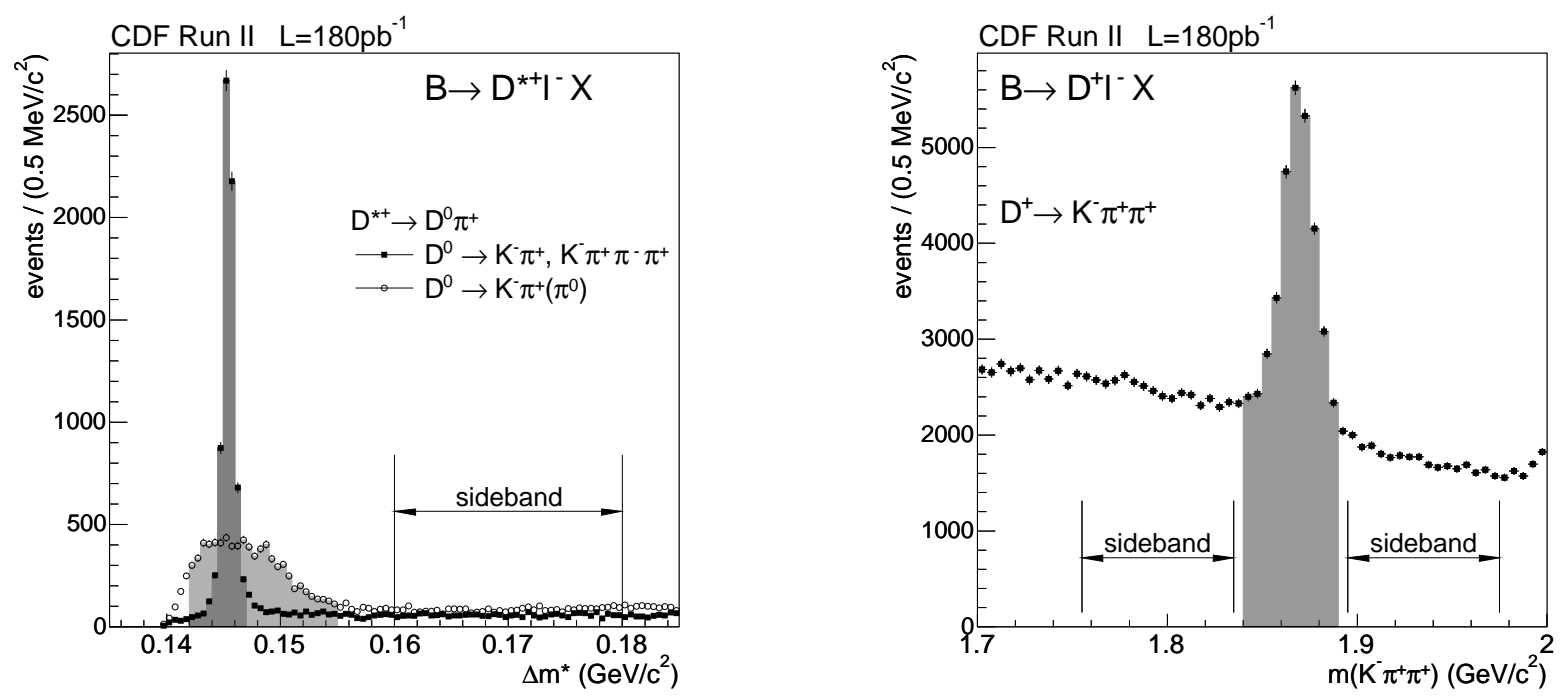

FIG. 1: Left: Mass difference $\Delta m^{*}=M\left(D^{0} \pi_{*}^{+}\right)-M\left(D^{0}\right)$ in the $D^{0} \rightarrow K^{-} \pi^{+}$and $D^{0} \rightarrow K^{-} \pi^{+} \pi^{+} \pi^{-}$channels $($narrow peak) and $\Delta m^{*}=M\left(K^{-} \pi^{+} \pi_{*}^{+}\right)-M\left(K^{-} \pi^{+}\right)$in the $D^{0} \rightarrow K^{-} \pi^{+} \pi^{0}$ channel (broad peak). Right: Mass distribution for the $D^{+} \rightarrow K^{-} \pi^{+} \pi^{+}$channel. The signal areas are shaded; sideband regions are also indicated.
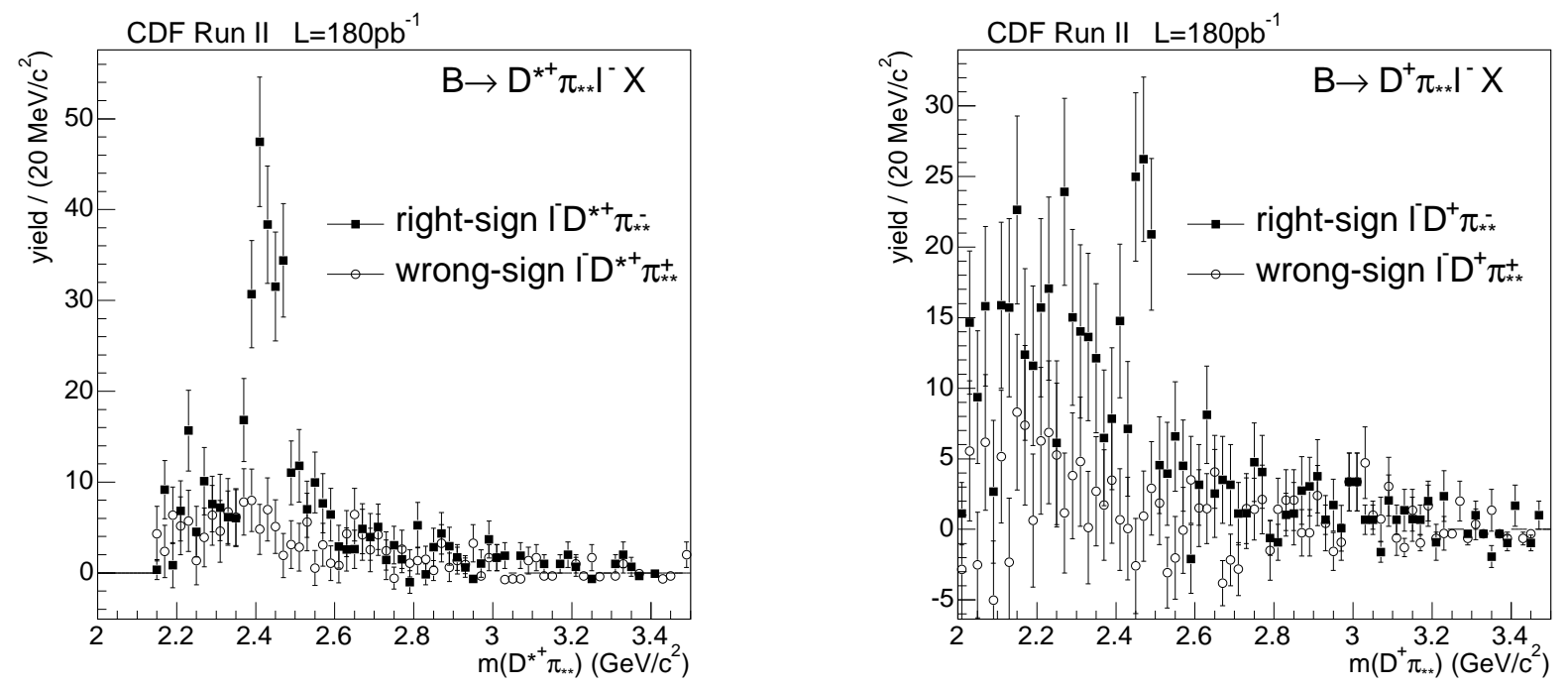

FIG. 2: Sideband-subtracted invariant mass distribution for the $D^{*+} \pi_{* *}$ channels (left) and for the $D^{+} \pi_{* *}$ channel (right). The mass regions shown are limited at $3.5 \mathrm{GeV} / c^{2}$ for illustration only. No explicit mass cut is applied in the analysis.

effectively removes any such background. Finally, the background from $B \rightarrow D^{(*)} \pi \tau \nu_{\tau}$ decays has been studied with Monte Carlo and found to have a negligible effect on the determination of the moments.

Since only the shape of the mass-squared distribution for the $D^{* *}$ component $\left(f^{* *}\left(s_{H}\right)\right.$ in Eq. (2) $)$ is being measured, the relevant efficiency corrections are those that can bias the mass-squared distribution, along with the relative efficiency for the $D^{*+}$ and $D^{+}$components of the $D^{* *}$ piece. Both efficiencies are obtained, as a function of the mass $M\left(D^{* *}\right)$, from the Monte Carlo simulation. We have checked the Monte Carlo relative efficiency predictions as a function of the $\pi_{* *}$ transverse momentum by applying the $\pi_{* *}$ selection cuts to decay tracks from $D$ and $D^{*}$ mesons. The efficiency variation in the data agrees well with the simulation. Where small differences have been found, corrections have been derived from these Monte Carlo-data comparisons.

To compare the moments with theoretical predictions, they must be measured with a well defined cut on $p_{l}^{*}$, the lepton momentum in the $B$ rest frame. Since we do not attempt to measure the boost of the $B$, we cannot access $p_{l}^{*}$ directly in data. Instead, acceptance corrections are derived from Monte Carlo that turn our gradual trigger 
turn-on as a function of $p_{l}^{*}$ into a sharp threshold at $p_{l}^{*}=$ $0.7 \mathrm{GeV} / c$, thereby correcting our measurement of the moments to a cut $p_{l}^{*}>0.7 \mathrm{GeV} / c$. The value $0.7 \mathrm{GeV} / c$ was chosen in order to minimize the correction. Because of the negative correlation between lepton momentum in the $B$ rest frame and $D^{* *}$ mass, the correction itself can depend on the detailed $D^{* *}$ mass spectrum in Monte Carlo. In order to assess the possible systematic error, the default $B$ decay model has been compared to a naïve phase-space $B$ semileptonic decay model. The differences in the ensuing correction factors as a function of $M\left(D^{* *}\right)$ are considered as systematic errors.

\section{RESULTS}

The $D^{(*)+} \pi^{-}$mass distribution is shown in Fig. [3 after background subtraction and efficiency and acceptance corrections. The first and second moments of the $D^{* *}$

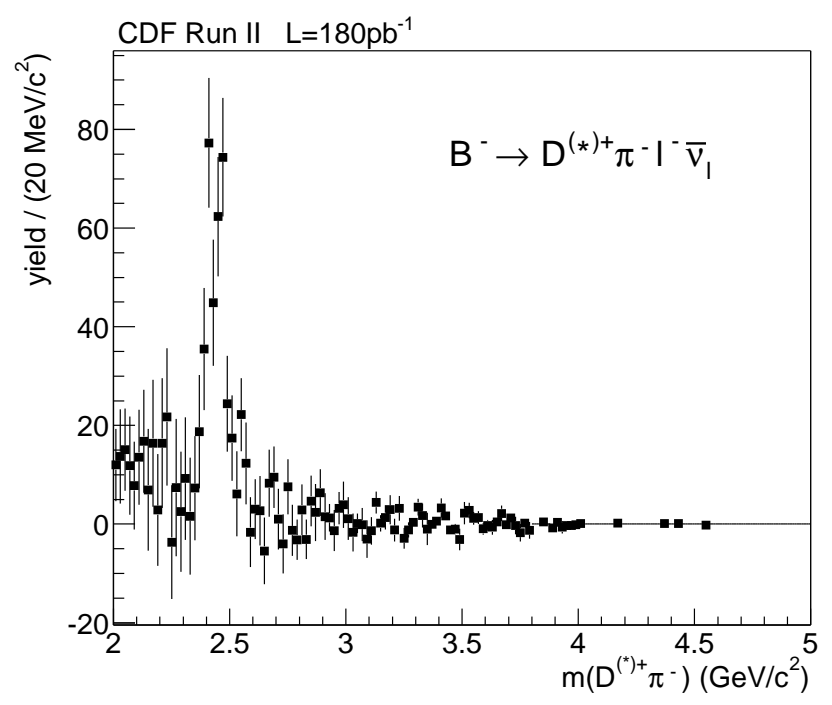

FIG. 3: Fully corrected invariant mass distribution $m\left(D^{(*)+} \pi^{-}\right)$. The number of events in each bin has been background subtracted and corrected for mass-dependent and $D^{*} / D^{+}$relative efficiency corrections. The plotted errors take into account all corrections and subtractions.

component of the mass-squared distribution, $m_{1}$ and $m_{2}$, are determined by simply computing the mean and variance of the distribution shown in Fig. 3. without any assumption about the shape or rate of its several components:

$$
\begin{aligned}
m_{1} \equiv\left\langle m_{D^{* *}}^{2}\right\rangle= & \left(5.83 \pm 0.16_{\text {stat }} \pm 0.08_{\text {syst }}\right) \mathrm{GeV}^{2} / c^{4} \\
m_{2} \equiv\left\langle\left(m_{D^{* *}}^{2}-\left\langle m_{D^{* *}}^{2}\right\rangle\right)^{2}\right\rangle= & \\
& \left(1.30 \pm 0.69_{\text {stat }} \pm 0.22_{\text {syst }}\right) \mathrm{GeV}^{4} / c^{8}
\end{aligned}
$$

with a $61 \%$ positive correlation. The full moments of the hadronic mass-squared distribution, $M_{1}$ and $M_{2}$, are determined by combining $m_{1}$ and $m_{2}$ with the $D$ and $D^{*}$ pieces, obtained from world-average values [3]:

$$
\begin{aligned}
M_{1} \equiv & \left\langle M_{X_{c}}^{2}\right\rangle-\bar{m}_{D}^{2}= \\
& \left(0.467 \pm 0.038_{\text {stat }} \pm 0.019_{\text {exp }} \pm 0.065_{\mathrm{BR}}\right) \mathrm{GeV}^{2} / c^{4} \\
M_{2} \equiv & \left\langle\left(M_{X_{c}}^{2}-\left\langle M_{X_{c}}^{2}\right\rangle\right)^{2}\right\rangle= \\
& \left(1.05 \pm 0.26_{\text {stat }} \pm 0.08_{\exp } \pm 0.10_{\mathrm{BR}}\right) \mathrm{GeV}^{4} / c^{8}
\end{aligned}
$$

with a $69 \%$ positive correlation between $M_{1}$ and $M_{2}$. Here "BR" refers to the uncertainty coming from the branching ratios needed for the combination of the $D$, $D^{*}$ and $D^{* *}$ pieces. For the exclusive branching ratios to $D$ and $D^{*}$, all available information [3] coming from charged and neutral $B$ decays has been combined using isospin invariance, leading to $\Gamma_{0} / \Gamma_{s l}=0.203 \pm 0.015$ and $\Gamma_{*} / \Gamma_{s l}=0.550 \pm 0.026$ with about $30 \%$ positive correlation. The isospin-related partial widths (not the branching ratios) of $B^{+}$and $B^{0}$ are assumed to be identical.

Finally, using the predictions in [2], the HQET parameters $\Lambda$ and $\lambda_{1}$ are determined. After applying constraints on the other HQET parameters coming from the known $B$ and $D$ hyperfine mass splittings we find, in the pole scheme: $\bar{\Lambda} \equiv \Lambda^{\text {pole }}=\left(0.397 \pm 0.078_{\text {stat }} \pm 0.027_{\exp } \pm\right.$ $\left.0.064_{\mathrm{BR}} \pm 0.058_{\text {theo }}\right) \mathrm{GeV}$ and $\lambda_{1}^{\text {pole }}=(-0.184 \pm$ $\left.0.057_{\text {stat }} \pm 0.017_{\exp } \pm 0.022_{\mathrm{BR}} \pm 0.077_{\text {theo }}\right) \mathrm{GeV}^{2}$, with a $79 \%$ negative correlation. Similarly, we extract the equivalent HQET parameters in the $1 \mathrm{~S}$ scheme: $m_{b}^{1 S} \equiv$ $M_{\Upsilon} / 2-\Lambda^{1 S}=\left(4.654 \pm 0.078_{\text {stat }} \pm 0.027_{\exp } \pm 0.064_{\mathrm{BR}} \pm\right.$ $\left.0.089_{\text {theo }}\right) \mathrm{GeV}$ and $\lambda_{1}^{1 S}=\left(-0.277 \pm 0.049_{\text {stat }} \pm 0.017_{\exp } \pm\right.$ $\left.0.022_{\mathrm{BR}} \pm 0.094_{\text {theo }}\right) \mathrm{GeV}^{2}$, with a $77 \%$ positive correlation.

The statistical and systematic errors in the extraction of the $D^{* *}$ moments, the full moments, and the HQET parameters in the pole-mass scheme are presented in Table I. Statistical errors dominate the measurements of $m_{1}$ and $m_{2}$ while experimental systematic errors are all smaller. The main experimental systematics are computed from the differences in the results when we apply or omit a correction for the $\sim 60 \mathrm{MeV} / \mathrm{c}^{2}$ mass resolution in the $K^{-} \pi^{+} \pi^{0}$ channel or the $M\left(D^{* *}\right)$-dependent efficiency correction from data. Similarly, we considered the difference in results obtained using Monte Carlo efficiency and acceptance corrections calculated with ISGW2 and Goity-Roberts matrix elements or phase space as a systematic error. Other experimental systematics include uncertainties on the level of the prompt background (studied with a fully reconstructed $B$ sample), a possible bias due to having used the same data sample to model the background in the optimization process and to subtract the background from the data (studied by repeating the optimization on bootstrap copies of the data), and uncertainties on $D$ branching fractions used in the analysis.

Uncertainties in the inclusive and exclusive semileptonic $B$ branching ratios become important when combining $m_{1}$ and $m_{2}$ with the $D^{0}$ and $D^{* 0}$ pieces to obtain $M_{1}$ and $M_{2}$, the moments of the entire charm mass distribution. Theoretical uncertainties become dominant in the extraction of the HQET parameters $\Lambda$ and $\lambda_{1}$. The 


\begin{tabular}{|l|c|c|c|c|c|c|}
\hline Error & $\begin{array}{c}\Delta m_{1} \\
\left(\mathrm{GeV}^{2} / c^{4}\right)\end{array}$ & $\begin{array}{c}\Delta m_{2} \\
\left(\mathrm{GeV}^{4} / c^{8}\right)\end{array}$ & $\begin{array}{c}\Delta M_{1} \\
\left(\mathrm{GeV}^{2} / c^{4}\right)\end{array}$ & $\begin{array}{c}\Delta M_{2} \\
\left(\mathrm{GeV}^{4} / c^{8}\right)\end{array}$ & $\begin{array}{c}\Delta \bar{\Lambda} \\
(\mathrm{GeV})\end{array}$ & $\begin{array}{c}\Delta \lambda_{1} \\
\left(\mathrm{GeV}^{2}\right)\end{array}$ \\
\hline Statistical & 0.16 & 0.69 & 0.038 & 0.26 & 0.078 & 0.057 \\
Total systematic & 0.08 & 0.22 & 0.068 & 0.13 & 0.091 & 0.082 \\
\hline \hline Mass resolution & 0.02 & 0.13 & 0.005 & 0.04 & 0.012 & 0.009 \\
\hline Efficiency (data) & 0.03 & 0.13 & 0.006 & 0.05 & 0.014 & 0.011 \\
\hline Efficiency and acceptance (MC) & 0.06 & 0.05 & 0.016 & 0.03 & 0.017 & 0.006 \\
\hline Background scale & 0.01 & 0.03 & 0.002 & 0.01 & 0.003 & 0.002 \\
\hline Background bias & 0.02 & 0.10 & 0.004 & 0.03 & 0.006 & 0.006 \\
\hline Physics background & 0.01 & 0.02 & 0.002 & 0.01 & 0.004 & 0.002 \\
\hline$D^{+} / D^{*+}$ branching ratios & 0.01 & 0.02 & 0.002 & 0.01 & 0.004 & 0.002 \\
\hline$D^{+} / D^{*+}$ efficiency & 0.02 & 0.03 & 0.004 & 0.01 & 0.005 & 0.002 \\
\hline$B$ semileptonic branching ratios & - & - & 0.065 & 0.10 & 0.064 & 0.022 \\
\hline$\rho_{1}$ & - & - & - & - & 0.041 & 0.069 \\
\hline$T_{i}$ & - & - & - & - & 0.032 & 0.031 \\
\hline$\alpha_{s}$ & - & - & - & - & 0.018 & 0.007 \\
\hline$m_{b}, m_{c}$ & - & - & - & - & 0.001 & 0.008 \\
\hline Choice of $p_{l}^{*}$ cut & - & - & - & - & 0.019 & 0.009 \\
\hline
\end{tabular}

TABLE I: Statistical and systematic uncertainties in the measurements of the $D^{* *}$ and full moments and in the extraction of $\bar{\Lambda}$ and $\lambda_{1}$ in the pole scheme.

largest contribution to the theoretical systematic error is that estimated by varying the unknown third order HQET parameters in the ranges $\rho_{1}=\frac{1}{2}(0.5 \mathrm{GeV})^{3} \pm$ $\frac{1}{2}(0.5 \mathrm{GeV})^{3}, T_{i}=(0.0 \mathrm{GeV})^{3} \pm(0.5 \mathrm{GeV})^{3}$. Finally, acceptance corrections have been computed for two alternative $p_{l}^{*}$ cuts, 0.5 and $0.9 \mathrm{GeV} / c$. The moments obtained this way are different physical quantities and numerically different from those obtained for the default $p_{l}^{*}$ cut at $0.7 \mathrm{GeV} / c$. However, if HQET describes the data, they should all lead to compatible values of the HQET parameters. The three sets of parameters are found to be equal within errors. Differences between them have been considered as additional systematic uncertainties.

In summary, we have presented a measurement of the first two moments of the hadronic mass-squared distribution in semileptonic $B$ decays to charm by combining our measurement of the $D^{(*)+} \pi^{-}$mass spectrum with the known masses and branching ratios to $D l \nu$ and $D^{*} l \nu$ taken from the Particle Data Group compilation [3]. These channels together are assumed to fully account for the inclusive semileptonic decay width of $B$ mesons to charm. The moments are then used to extract the two leading Heavy Quark Effective Theory parameters, $\Lambda$ and $\lambda_{1}$, in both the pole mass and the $1 \mathrm{~S}$ mass schemes. Within HQET the results are in agreement with previous determinations at $e^{+} e^{-}$machines [10], mostly at higher values of $p_{l}^{*}$, and our precision in the moments is comparable or slightly better. The experimental techniques and systematic uncertainties are very different from those at electron-positron colliders. Our measurements, when combined with moments of the lepton energy in semilep- tonic B decays 11] and photon energy in $b \rightarrow s \gamma$ transitions [12] measured elsewhere, will help pinpoint the values of the non-perturbative HQET parameters [13]. This will sharpen the experimental determination of $\left|V_{c b}\right|$ and allow tests of the underlying assumptions of the theoretical framework, such as quark-hadron duality.

\section{ACKNOWLEDGMENTS}

We thank the Fermilab staff and the technical staffs of the participating institutions for their vital contributions. It is a pleasure to thank Zoltan Ligeti for several enlightening discussions regarding the HQET predictions. This work was supported by the U.S. Department of Energy and National Science Foundation; the Italian Istituto Nazionale di Fisica Nucleare; the Ministry of Education, Culture, Sports, Science and Technology of Japan; the Natural Sciences and Engineering Research Council of Canada; the National Science Council of the Republic of China; the Swiss National Science Foundation; the A.P. Sloan Foundation; the Bundesministerium für Bildung und Forschung, Germany; the Korean Science and Engineering Foundation and the Korean Research Foundation; the Particle Physics and Astronomy Research Council and the Royal Society, UK; the Russian Foundation for Basic Research; the Comisión Interministerial de Ciencia y Tecnología, Spain; and in part by the European Community's Human Potential Programme under contract HPRN-CT-2002-00292, Probe for New Physics. 
[2] C. W. Bauer, Z. Ligeti, M. Luke, A. V. Manohar, Phys. Rev. D 67, 054012 (2003).

[3] S. Eidelman et al., Phys. Lett. B592, 1 (2004).

[4] D. Acosta et al. (CDF Collab.), Phys. Rev. D68, 072004 (2003).

[5] B. Ashmanskas et al., Nucl. Instrum. Meth. A518, 532 (2004).

[6] D. Scora, N. Isgur, Phys. Rev. D 52, 2783 (1995).

[7] J. L. Goity, W. Roberts, Phys. Rev. D 51, 3459 (1995).

[8] R. Brun, F. Carminati, CERN Programming Library Long Write-up W5013 (1993).

[9] B. Efron, Ann. Stat. 7, 1 (1979); A. Stuart, J. K. Ord, Kendall's Advanced Theory of Statistics, Vol. 1, 5th Ed., (Oxford University Press, New York, 1987).
[10] D. Cronin-Hennessy et al. (CLEO Collab.), Phys. Rev. Lett. 87251808 (2001); M. Battaglia et al., Phys. Lett. B556, 41 (2003); B. Aubert et al. (BABAR Collab.), Phys. Rev. D 69, 111103 (2004).

[11] H. Mahmood et al., (CLEO Collab.), Phys. Rev. D 67, 072001 (2003); M. Battaglia et al., Phys. Lett. B556, 41 (2003); B. Aubert et al. (BABAR Collab.), Phys. Rev. D 69, 111104 (2004).

[12] S. Chen et al. (CLEO Collab.), Phys. Rev. Lett. 87, 251807 (2001).

[13] C. W. Bauer, Z. Ligeti, M. Luke, A. V. Manohar, M. Trott, Phys. Rev. D 70, 094017 (2004). 